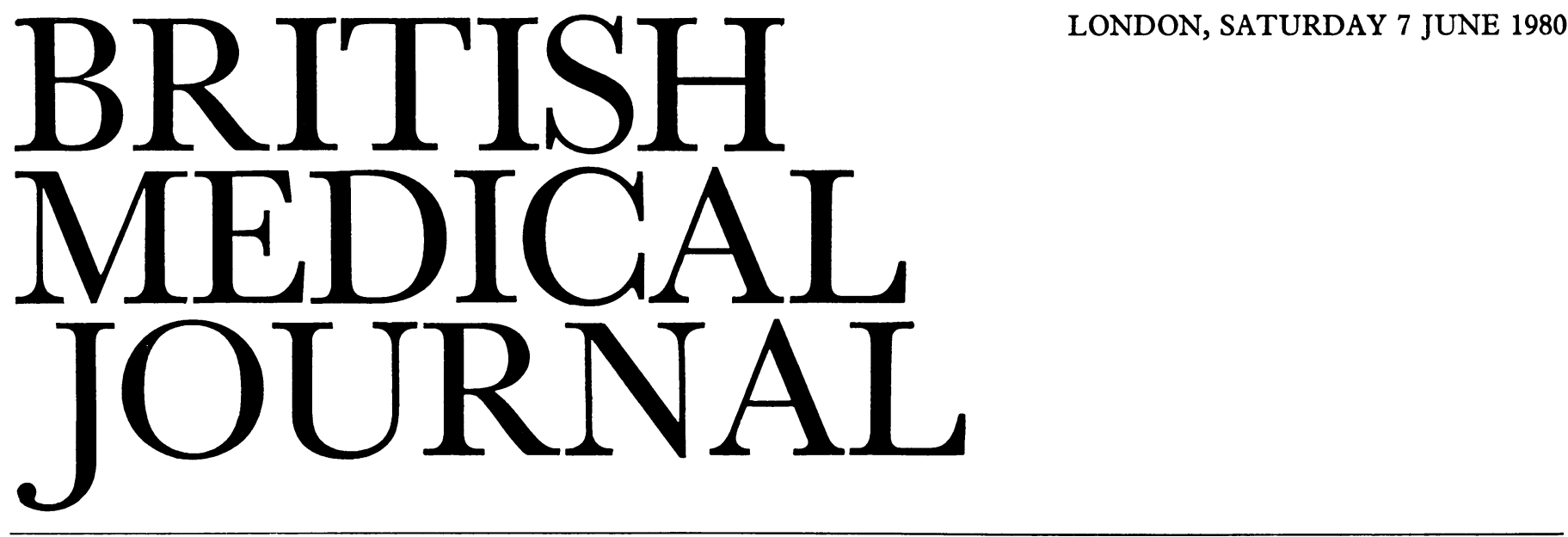

The chemical pathology department of an average district general hospital may report on over 500 blood urea estimations each day. In one year up to 1000 patients may have values over $16 \mathrm{mmol} / 1(100 \mathrm{mg} / 100 \mathrm{ml})^{12}$ so that each evening several house officers may be presented with such a result. The ability to assess the meaning of a raised blood urea concentration should be within the competence of any doctor ordering the investigation, but knowledge about renal dysfunction is increasingly sequestered in renal units. ${ }^{3}{ }^{4}$ Patients, however, do not recognise boundaries and may develop signs or symptoms of renal failure at any time and in any hospital. The immediate transfer to a renal unit of every uraemic patient is obviously impracticable, so that physicians, especially those working in isolation from renal units, need to keep up to date with nephrological practice.

In one typical study, ${ }^{2}$ based on a laboratory serving 750000 people, 60 cases of acute renal failure were identified in a year. The most common cause was acute tubular necrosis, followed by a mixed range of conditions including acute glomerulonephritis and myeloma and a scattering of disorders accounting for single examples. These 60 patients had to be distinguished from the 1500 with prerenal renal failure, the 200 or so with chronic renal failure, and the 150 with obstructive (postrenal) renal failure. These distinctions may sometimes be obvious, but in other cases this first stage in the classification of uraemia may be difficult, especially when prerenal factors complicate either acute or chronic renal failure. In uraemia of all types the need for rehydration or expansion of the blood volume must be recognised quickly, since specific treatment may be life saving in some patients and in others may improve the clinical condition sufficiently to make the diagnosis of prerenal renal failure certain.

Clinical examination is vital both initially and then subsequently during treatment. These clinical assessments must not be replaced by excessive reliance on the laboratory service, or by the monitoring services provided in many intensive care units. Examination of a patient in renal failure should start by determining the central venous pressure clinically from the deep jugular venous pressure (not only with the patient at $45^{\circ}$, but at whatever angle seems appropriate). The experienced clinician evaluates the arterial circulation by taking the blood pressure at different angles, determining if there is a postural drop, and he studies the circulation of the nose, the ear lobes, and the digits. He searches for information about acute changes in weight - a much better guide to fluid balance than most fluid balance charts-and he is more impressed by clinical signs of oedema than by paper evidence of a negative fluid balance. He may measure the specific gravity of the urine and go to the nearest microscope to study the deposit. The enthusiastic "do it yourself" man may have his own kit to measure the urinary chloride concentration in the side room (as an alternative to the urinary sodium concentration, which remains a laboratory test). Measuring the venous pressure by a central catheter may be needed as an aid to clinical evaluation, and measurement of the osmolality and electrolyte content of samples of urine and plasma collected during the acute phase of the illness and before active intervention can give useful clues.

A prerenal problem should usually be obvious, or at least should be suspected, once the clinician has taken a history, inspected the records, and examined the patient. If there is no acute or chronic renal disease and uraemia is just prerenal plasma concentration of urea will usually show a greater rise in proportion to the normal range than will the creatinine; indeed, a plasma concentration of $>250 \mu \mathrm{mol} / 1$ has a $90 \%$ probability of being associated with renal impairment. ${ }^{5}$ Urinary concentrations of sodium and chloride are usually low in prerenal failure, while the specific gravity and osmolality are higher than isotonic. Patients with chronic renal disease complicated by heart failure or hypoproteinaemia and some with acute renal disorders (especially if there are disturbances of blood supply, in hepatic failure, and in acute glomerulonephritis) may pass urine similar to that from patients with prerenal failure, having a low sodium chloride content. If glomerular filtration is much reduced the urine will have an osmolality more nearly isotonic with serum than when uraemia is prerenal and the kidneys are healthy. In patients with raised plasma concentrations of urea and creatinine discriminatory tests based on measurement of urinary constituents have been found to be imprecise, ${ }^{6}$ despite being recommended in many texts. A careful analysis of the contribution of urinary sodium, urinary osmolality, and urine:plasma creatinine ratio in a group of 87 patients with acute renal failure and serum creatinine concentrations over $180 \mu \mathrm{mol} / 1$ $(2 \mathrm{mg} / \mathrm{dl})$ has recently been reported by Espinel and Gregory. ${ }^{6}$ Urinary and plasma sodium and creatinine results (measured on "stat" samples) were used to calculate the fractional excretion of sodium (urinary $\mathrm{Na}$ /plasma $\mathrm{Na} \div$ urinary creati- 
nine/plasma creatinine $\times 100$ ). When expressed in this way patients with acute tubular necrosis, either oliguric or nonoliguric, and those with urinary obstruction had a fractional excretion greater than unity but in those with prerenal failure or acute glomerulonephritis the ratio was less than one. Only one patient in 87 was misclassified. The calculation should not be misapplied to patients with co-existing chronic renal impairment, interstitial nephropathy, or glycosuria or to those who have already had diuretics, in whom disturbances of fractional excretion of sodium could be for other reasons.

The most important diagnostic criterion of prerenal uraemia remains the response to treatment. Replacement of a deficit in the blood volume must take into account whether the deficit is only intravascular (hypoproteinaemia, for example), or also extracellular (sodium and water deficits) or a deficit in the total body water ("simple" water deficits). Treatment may be urgent and must be adequate and closely monitored. Fluids may need to be given at a rate of one to three litres an hour, or even faster, with the doctor standing by the infusion, rather than more slowly with the clinician elsewhere and unable to observe the response. In uncomplicated prerenal failure a response to treatment is usually obvious (but when there is cardiac or hepatic failure, such a test must be used with extreme caution if at all). As soon as body fluid deficits are made up the flow of urine will increase and the plasma concentration of urea will fall. If uraemia is not just prerenal careful observation should avoid the danger of the excessive fluid replacement that can easily occur when deficits have been replaced but the kidney cannot respond to excesses.

A failure to respond to treatment for prerenal failure, or other indications that the illness may be renal or postrenal, raises the difficult question of judging the appropriate time for advice from a specialised unit. Investigation of many of these patients will require radiological studies and often also renal biopsy-both procedures which, when done to diagnose the cause of uraemia, lose some of their diagnostic value in the hands of those using the techniques infrequently. Similarly, the management of patients with hyponatraemia, hyperkalaemia, overhydration, and undernutrition ${ }^{7}$ is easier if the medical and nursing staff are experienced and have easy access to techniques such as haemodialysis and ultrafiltration. ${ }^{8}$

High-dose intravenous urography with tomography has been one of the major advances in diagnostic methods in the last two decades. ${ }^{910}$ In patients with renal failure retrograde urography is now reserved for the preoperative evaluation of a few selected patients with postrenal obstruction already diagnosed by intravenous urography. Antegrade urography after direct needle puncture of the renal pelvis is often a more satisfactory radiological technique than retrograde examination. Radiological investigation has considerable diagnostic potential $^{1112}$ and is much safer and effective in patients with raised concentrations of urea than used to be thought. There has recently been some renewed uncertainty about the place of the technique, especially in diabetics, ${ }^{13}$ but in acute renal failure the advantages of having good information usually outweigh any potential hazards. The contribution of radiology was clearly shown in the index case of a recent series of patients with acute renal failure from acute pyelonephritis. ${ }^{11}$ The diagnosis of acute renal failure in that case was first suspected at intravenous urography and only later confirmed by biochemical tests. The same paper showed the limitations of even the best radiological studies when the disorder is renal rather than postrenal. Postrenal failure can be excluded and a specific disorder affecting the renal parenchyma suspected, but few of the renal disorders presenting as acute renal failure have pathognomonic radiological features allowing a definite diagnosis. In four of the patients with suspected acute pyelonephritis who survived some diagnostic uncertainty remained because no pathological specimens were available. Only in the patient who died, and had a necropsy, was there no possible doubt.

Diagnostic uncertainty is unsatisfactory in case reports but may be justified when certainty is not needed to manage the patient. What is quite unacceptable is for a patient with renal failure to remain undiagnosed with clinical and initial urographic findings suggesting several possible alternatives needing different treatment. In some such cases further radiological studies of the renal arteries, veins, or the collecting system and ureters may be a more appropriate next step, but in others renal biopsy may be needed.

With ultrasound or urographic localisation of the kidney, and with gel-foam embolism available to arrest the rare renal haemorrhage, ${ }^{12}$ renal biopsy is now very safe. In many cases of acute renal failure biopsy should be done soon after the initial assessment has excluded prerenal and postrenal causes and after correction of prerenal aspects. Immuno-microscopical and electron-microscopical studies should be arranged as well as conventional histological examinations. The many other investigative techniques now available may provide little extra useful information at considerable cost. Some, like those for systemic lupus erythematosus, can provide important clues to a disease needing urgent treatment but do not often remove the need for renal biopsy.

Transfer to units with specialised facilities should be considered early rather than late, especially when there is no local expert to advise. Transfer may be needed for diagnosis; but even when the diagnosis is certain the timing of transfer for dialysis can be difficult. Plotting sequential plasma concentrations of creatinine against time may be useful: a straight line plot often results, especially if the weight-corrected reciprocal of creatinine is used, ${ }^{14}{ }^{15}$ and extrapolation will then allow the physician to predict when dialysis will be needed. A change in the slope suggests improvement, either spontaneous or from treatment. If there are no clear indications of a diuresis with improvement of renal function treatment can be planned. Few nephrologists feel comfortable observing plasma concentrations of urea of over $30 \mathrm{mmol} / 1(180 \mathrm{mg} / 100 \mathrm{ml})$, and extrapolation into this area is a reason to plan dialysis. Many patients in acute renal failure receive peritoneal dialysis, though most nephrologists, if they were found to be in renal failure, would prefer haemodialysis, and peritoneal dialysis in the hands of the inexpert can be lethal.

The differential diagnosis of acute renal failure needs skill; each year more causes are added to the already lengthy textbook lists. It often takes considerable thought, with experience being a useful adjunct, to separate out the many possible causes, some of which need specific treatment. The clinician may need to ask himself whether he is not working too close to the limits of what he knows. Is there someone else who works on the next floor, or at another hospital, whose experience might be critical to the survival or the efficient management of his patient in renal failure ? Uraemia is common, but diagnosis and treatment are increasingly complex-yet can be straightforward when there is an experienced group of experts with adequate facilities.

${ }^{1}$ Branch RA, Clark GW, Cochrane AL, Jones, JH, Scarborough H. Incidence of uraemia and requirements for maintenance haemodialysis. Br Med F 1971;1:249-54.

${ }^{2}$ Dombey SL, Sagar D, Knapp MS. Chronic renal failure in Nottingham and requirements for dialysis and transplant facilities. $\mathrm{Br} M e d \mathcal{F ~ 1 9 7 5 ; ~}^{2}$ 2:484-5. 
${ }^{3}$ Kennedy AC, Burton JA, Luke RG, et al. Factors affecting the prognosis in acute renal failure. $Q \mathcal{F}$ Med $1973 ; 42: 73-86$

${ }^{4}$ Kerr DNS. Acute renal failure. In: Black D, ed. Renal disease, 3rd ed. Oxford: Blackwell Scientific Publications, 1972:417-61.

5 Morgan DB, Carver ME, Payne RB. Plasma creatinine and urea: creatinine ratio in patients with raised plasma urea. $\mathrm{Br} M e d \mathcal{f} 1977 ; 2: 929-32$

${ }^{6}$ Espinel CH, Gregory AW. Differential diagnosis of acute renal failure. Clin Nephrol 1980;13(2):73-7.

7 Cattell WR. In: NF Jones, ed. Recent advances in renal disease: Acute renal failure. London: Churchill Livingstone, 1975:1-47.

${ }^{8}$ Bergström J, Asaba H, Furst P, Oulés R. Dialysis, ultrafiltration and blood pressure. Proc Eur Dial Transplant Assoc. 1976;13:293-300.

${ }^{9}$ Fry IK, Cattell WR. In: Black D, Jones NF, eds. Renal disease, 4 th ed. Oxford: Blackwell Scientific Publications, 1979.

${ }^{10} \mathrm{McClennan} \mathrm{BL}$. Current approaches to the azotaemic patient. Radiol Clin North Am 1979;17:197-211.

11 Baker LRI, Cattell WR, Fry IKF, Mallinson WJW. Acute renal failure due to bacterial pyelonephritis. $Q \mathcal{F}$ Med $1979 ; 48: 603-12$

12 Allison DJ. Therapeutic embolization. Br f Hosp Med 1978;20:707-14.

${ }^{13}$ Harkonen S, Kjellstrand CM. Exacerbation of diabetic renal failure following intravenous pyelography. $A m \mathcal{F}$ Med 1977;63:939-46.

${ }^{14}$ Rutherford WE, Blondin J, Miller JP, Greenwatt AS, Vavra JD. Chronic progressive renal disease: rate of change of serum creatinine concentration. Kidney Int 1977;11:62-70.

${ }^{15}$ Knapp MS, Blamey R, Cove-Smith R, Heath M. Monitoring the function of renal transplants. Lancet $1977 ; \mathrm{ii}: 1183$.

\section{Making a virtue of necessity}

After reading Hospital Services: the Future Pattern of Hospital Provision in England ${ }^{1}$ health authorities may not be much the wiser about what to do with the plans for their long-awaited 800-bed district general hospitals. The paper, recently issued by the Minister for Health, Dr Gerard Vaughan, marks an official move away from the concentration of hospital services on to one site that started with the Hospital Plan of $1962^{2}$ and developed throughout the 'sixties. ${ }^{3}{ }^{4}$ It proposes that centralisation should now give way to dispersal "to allow for the retention of a wider range of local facilities."

Though Hospital Services is officially a consultation document, Dr Vaughan has already reviewed 66 plans in the light of it (he approved 50). Indeed, in announcing his paper, $\mathrm{Dr}$ Vaughan seemed unsure whether it represented a major change in policy or merely a sensitive tinkering to reflect the financial climate and current disenchantment with large institutions. The policy of the 'sixties has not in fact produced great concentration: many district general hospitals are a mixture of old and new buildings on several sites, over half of England's hospitals have fewer than 100 beds, and only about 30 general hospitals have more than 800 . Health authorities have had to modify plans for centralised hospitals (or never made them) simply because the money never matched their aspirations.

The arguments against large hospitals and in favour of small ones outlined in Dr Vaughan's paper will be familiar to those who have tried to close small hospitals in the face of local opposition: large hospitals are expensive to run, difficult to manage, hard to get to, and impersonal; smaller hospitals are easier to staff, more personal, easier to get to, and derive more support from their community. While emphasising the need for different patterns of service to meet different needs, the paper specifically recommends a main hospital of 450 to 600 beds (with a further 200 or so in teaching hospitals or for regional specialties) with an accident and emergency service, medical and surgical beds, the children's unit, some maternity beds, a small psychiatric unit, and $30 \%$ of the geriatric beds. Local hospitals might provide geriatric and psychiatric services, some outpatient clinics, day surgery, and a casualty service "preferably run by general practitioners."

Hospital Services acknowledges that a review of policy was necessary for financial reasons-health authorities will have to maintain existing facilities because there is little capital to reorganise them-but it does recognise some of the difficulties of dispersal. These include the expense of running two hospitals rather than one, the greater costs of medical staffing, and the problem of deploying and training nurses: "Such a policy could therefore result in a lower overall quantity of clinical service for a given level of revenue than would be possible with a more concentrated service." Such a policy is unlikely to be welcomed by authorities trying to maintain levels of service with less money than before. At best Dr Vaughan's paper merely recognises what health authorities are already doing in the face of lack of capital and delayed plans. At worst it is a dishonest response to the real problem of hospital provision in England: a persisting lack of capital investment in the NHS.

1 Department of Health and Social Security. Hospital services: the future pattern of hospital provision in England. London: DHSS, 1980.

${ }^{2}$ Ministry of Health. $A$ hospital plan for England and Wales. London: HMSO, 1962. (Cmnd 1604).

${ }^{3}$ Ministry of Health. The hospital building programme: a revision of the hospital plan for England and Wales. London: HMSO, 1966. (Cmnd 3000)

${ }^{4}$ Central Health Services Council. The functions of the district general hospital. London: HMSO, 1969.

\section{Genital herpes}

Genital herpes has recently been described as the most important sexually transmitted disease. ${ }^{1}$ It has a higher prevalence than other sexually transmitted diseases and a tendency to recur; we have no effective treatment or vaccine; and there is an association between recurrence and sexual problems and a link with subsequent carcinoma of the cervix. Sexual transmission can take place in the absence of symptoms or signs, and a baby may be infected during delivery, sometimes with catastrophic results, though this seems to be a more of a problem in the United States than in Britain.

Gardner found that genital herpes accounted for $13.5 \%$ of cases of all sexually transmitted disease in his private practice in Houston, Texas. ${ }^{1}$ Figures for Britain are hard to obtain: only the cases seen in clinics for the treatment of sexually transmitted diseases are notified, and many must be seen elsewhere without notification. One survey ${ }^{2}$ suggests that in about $60 \%$ of cases notified from these clinics the diagnosis is soundly based on clinical observation supported by the results of viral culture; but in units such as dermatology and gynaecology departments the diagnosis is less likely to be supported by the results of viral culture.

Genital herpes was first notified as a separate disease by the sexually transmitted disease clinics in Britain in 1971, when 3671 new cases were recorded, or $12 \cdot 2$ per 100000 population. $^{3}$ By 1976 the total had doubled, to 7547; and by 1978 the provisional total was 8957 , or 28.8 per 100000 population. $^{4}$ The disease is now about twice as common as syphilis, for which the provisional number of new cases (of all forms) was 4802 in $1978 .{ }^{4}$ Genital herpes is thus much commoner than primary syphilis, from which it must be differentiated; the clinics report about one fresh case of primary syphilis 